\title{
The Application of Scales in the Diagnosis of Perceived Needs in the Elderly with Mild Cognitive Impairment
}

\author{
Josélia Braz dos Santos Ferreira1, Selma Petra Chaves Sa르, Luciana Krauss Rezende², \\ Rosana Moreira de Sant'Anna1, Ana Maria Domingos ${ }^{3}$, Rosimere Ferreira Santana² \\ ${ }^{1}$ University Hospital Antônio Pedro, Universidade Federal Fluminense (UFF), Niterói, Brazil \\ ${ }^{2}$ Afonso Aurora Costa Nursing School, Universidade Federal Fluminense (UFF), Niterói, Brazil \\ ${ }^{3}$ Ana Neri School of Nursing, Universidade Federal do Rio de Janeiro (UFRJ), Rio de Janeiro, Brazil \\ Email: joseliabraz42@yahoo.com.br, spetra@ig.com.br, anamaria_domingos@yahoo.com.br, \\ rosifesa@gmail.com
}

Received 6 July 2016; accepted 21 August 2016; published 24 August 2016

Copyright (C) 2016 by authors and Scientific Research Publishing Inc.

This work is licensed under the Creative Commons Attribution International License (CC BY). http://creativecommons.org/licenses/by/4.0/

(c) (i) Open Access

\section{Abstract}

The study evaluated the use of the Mini-Mental State Examination scale (MMSE), Tinettiscale, and Motor Scale for the Elderly (EMTI) toassist in the diagnosis of potential needs observed in elderlies with Mild Cognitive Impairment. This was aquasi-experimental research, conducted in a Basic Health Unit in thecityof Rio de Janeiro in 2014. The sample population consisted of 22 elderlies aged 64 to 88 years and $86.36 \%$ females. The SAS statistical software (version 9.3.1) and KruskalWallis test were used at a $95 \%$ confidence interval and a significance level of 0.05 and demonstrated significant differences in the evaluations performed before and after the intervention. The detected diagnoses were: impaired memory, the risk of falls, and willingness to improved relationships, among others. The evaluations showed MMSE results that were suggestive of cognitive impairment in $22.73 \%$ of the elderlies; the Tinetti scale showed a high risk of falls in $31.82 \%$ of theelderlies; and EMTI with 88.36 points, which was equivalent to the normal low classification. The intervention took place through ten weekly activity sessions after the initial evaluations. In the second evaluation, the Tinetti showed $59.09 \%$ of the elderlies with a moderate risk of falls and the EMTI as the normal average classification with $\mathbf{9 0 . 3 2}$ points. It was concluded that the scales offered diagnostic possibilities, which allowed for the implementation of necessary interventions according to the detected problems.

\section{Keywords}

Nursing Diagnosis, Mild Cognitive Impairment, Sensory-Motor Performance, Postural Balance, Elderlies

How to cite this paper: Ferreira, J.B.S., Sa, S.P.C., Rezende, L.K., de Sant'Anna, R.M., Domingos, A.M. and Santana, R.F. (2016) The Application of Scales in the Diagnosis of Perceived Needs in the Elderly with Mild Cognitive Impairment. Open Journal of Nursing, 6, 573-581. http://dx.doi.org/10.4236/ojn.2016.68061 


\section{Introduction}

Increasing longevity is a worldwide phenomenon. The proportion of elderlies is growing rapidly in Brazil and in many countries, with demographic projections suggesting that the age group above 65 years should increase from 14.9 million ( $7.4 \%$ of total) in 2013 to 58.4 million (26.7\% of total) in 2060 [1]. Although longer life expectancy may be considered an achievement, it is associated with an increased incidence of chronic degenerative diseases, which leads to a significant growth in demand for health services. Many elderlies maintain high levels of cognitive functioning throughout life [2], however, many present some decline in some cognitive abilities. The increasing number of elderly patients with dementia using public health services deserves special attention, especially if the progression trend continues and this chronic degenerative disease assumes epidemic proportions. Because there are few alternative therapies and treatments with the early diagnosis are more effective, it is essential to identify cases that present a high risk of progression to dementia [3]. A program of activities implemented by a multidisciplinary team can contribute significantly to life's structure and cognitive function in the elderly population [4].

The concept of Mild Cognitive Impairment (MCI) can be defined as impairment revealed in changes in memory, a situation which does not imply dementia. Tests such as the Mini-Mental State Examination (MMSE) are used to diagnose MCI. Criteria for this diagnosis include normal general cognitive functions and socio-occupational activities and a minimum score of 24 points (performance in about 1.5 standard deviation below the mean of normal controls of same age and education level) [3] [5] [6].

The assessment of functional capacity is necessary to know the degree of dependence/independence in the elderly [7], which represents the independence an individual has to perform physical and mental activities necessary to maintain basic and useful activities [8]. Longevity, when associated with non-transmissible chronic diseases, can affect the functional capacity of elderlies and thereby prevent them from performing independent activities and fostering dependence on another person [9].

Therefore, early screening of cognitive and functional conditions in the elderly is key to improving life structure and cognitive function in the elderly population.

Nurses play an important role in promoting health and preventing disease in the elderly population, especially in the context of cognitive health. Therefore, it is essential that nursing evaluations are used to determine appropriate interventions.

Hence, NANDA International [10] offers proposals for specific nursing diagnoses as well as interventions commonly associated with those diagnoses. These interventions correspond to activities necessary for the determination and implementation of the assistance required for each client and may be used in different contexts.

Nursing evaluations using specific scales for the elderly, as is the case in this study, are important in relating a diagnosis with the appropriate intervention(s). Nursing evaluations provide a systematic and dynamic method of providing humanized care, which is oriented to obtain the best results [11].

This study used the Mini-Mental State Examination (MMSE), Motor Scale for the Elderly (EMTI), and Tinetti scale to assist in the diagnosis of perceived needs in elderly patients with mild cognitive impairment to achieve care goals.

\section{Methods}

This quasi-experimental study was conducted over four months. Twenty-two male and female elderlies over 60 years of age who were located in Rio de Janeiro and users of a Basic Health Unit (BHU) participated. The study was conducted in the outpatient clinic of the institution; the participants attended the Memory Workshop.

Medical records of elderly participants indicated mild cognitive impairment as defined by the researcher. Diagnosis of cognitive impairment was based on examinations such as magnetic resonance, computerized tomography, and laboratory tests among others. Referrals made by the institution's geriatric assessment were also considered.

Inclusion criteria were: males and females over 60 years of age with Mild Cognitive Impairment (MCI) and a score above 24 on the Mini-Mental State Examination for individuals with higher education, at least 18 for individuals with high school education, or above 14 for the illiterate. Exclusion criteria were individuals who missed $25 \%$ of the psychomotor stimulation activities, had severe vision deficit, presented recent bone injuries, had prosthetic lower limbs, had a surgical procedure in the last 6 months, were affected with labyrinthitis, and who 
had hemodynamically unstable cardiovascular disease.

As the number of elderly, in principle, to obtain a significant number a survey was conducted in this institution. According to surveys conducted in the clinic of the Municipal Hospital of Geriatrics and Gerontology of Rio de Janeiro Health (Brazil) in the period from September to November 2013 were attended 679 elderly. Patients with Mild Cognitive Deficit are directed to cognitive stimulation, already existing in service 57 elderly.

Using this information, and a significance level of 0.05 , standard error of 0.15 percent, it has to be for this institution the sample is at least 13 elderly in order to have a significant sample. These are the minimum values to make the sample representative for the institution and so there is the possibility of evaluating these elderly and perform interventions in time for the research. A total of 34 elderlies were initially evaluated: 12 (35.3\%) were excluded through the exclusion criteria and 22 (64.7\%) participated were included in the study outcomes, which were also considered a significant sample number based onaninitial study survey.

Instruments used included: the Mini-Mental State Examination-MMSE [12] [13]; Tinetti Scale [14]; and Motor Scale for the Elderly-EMTI [15]. They were used to determine psychomotor activities after diagnosis; activities were designed to promote balance and functional capacity. Group activities were conducted for ten weekly sessions. The Tinetti [14] and EMTI [15] scales were applied at the beginning of the study and at the end of the performed psychomotor stimulation program.

The Mini-Mental State Examination [12] [13], which is a cognitive impairment screening mechanism was used in this study only the first step to evaluate the cognition of the participants. In this case, considering the scale EMTI complete by cover all psychomotor factors, the good results on this scale corresponds also to improvement in cognition in the elderly.

This instrument was developed by Folstein and McHugh [12] and translated by Bertolucci et al. [13] to assess specific cognitive functions: time orientation (5 points), location orientation (5 points), three-word record (3 points), attention and calculation (5 points), recall of the three words (3 points), language (8 points), and visual-constructive capacity (1 point). The scoring system ranges from zero to 30 . In this study, the validated version used the following cutoff points: 13 for illiterates, 18 for medium and low education levels, and 26 for higher education level [13]. The Tinetti [14] and EMTI [15] Scales were used for diagnostic evaluation before and after the intervention.

The validated version of the Tinetti scale is an instrument that utilizes the Mary Tinetti protocol [14]. This protocol predicts the risk of falls by detecting changes in gait and diagnoses and quantifies the severity of impairment. According to this instrument, the lower the score is, the greater the problem is. A score lower than 19 indicates five times higher risk of falls. Thus, individuals who presented scores below 19 points present a high risk of falls; those between 19 and 24 a moderate risk; and those over 24 do not present a risk of falls.

The Tinetti index consists of two scales: balance with a total of 16 points and gait with a total of 12 points (total points: 28). The balance component consists of 9 items: sitting balance, rising from a chair, attempts to rise, balance after rising (first 5 seconds), standing balance, the three times tests, eyes closed, balance while turning 360 degrees, and balance while sitting. In the tasks requiring the use of a chair, the patient starts the evaluation in a rigid chair without armrests and with his back in the upright position [14].

The gait has seven items: gait start, length and height of steps, steps' symmetry, continuity of steps, direction, trunk, and ankles' distance. In the tasks/maneuvers in which the use of a chair is needed, the patient starts the evaluation in a rigid chair without armrests, and with his back in the upright position [14].

The Motor Scale for the Elderly-EMTI [15] is an innovative, validated instrument [16], which provides new opportunities for evaluation and intervention in the elderly population. Evaluation using motor tests allows verification of the progressive stages of physical function and detection of the decline in physical parameters and, in turn, provides for the planning of effective intervention strategies. This is an exploration method that evaluates specific areas of human motricity (body stability and proprioception movements).

Each test has different degrees of difficulty that are presented in an increasingly progressive order. The scores obtained in the evaluation allow the classification of motor parameters in levels: very high (130 or above), high (120 129), normal high (110 - 119), normal average (90 - 109), normal low (80 - 89), low (70 - 79), and very low (<70).

Interventions: elderlies could miss only two out of the ten sessions. Stimulating cognitive and psychomotor activities to improve psychomotor quality through an exercise program were developed according to NANDA-I [10] after diagnosis. Scales were used and followed-up by the researcher. Dynamics suggested by Rosa Neto [15] were used with supplemental music and videos according to the theme. Ten weekly group exercising activities were conducted for a period of four months. The participants were re-evaluated with the Tinetti and EMTI scales at the end of these sessions. 
SAS statistical software version 9.3.1was used for data analysis. Data were divided into initial and final evaluations, i.e., before the start of activities and after the end of activities. The initial descriptive analysis included all 22 elderlies.

The Shapiro-Wilks normality test was used. Because variables were not normally distributed, the signal nonparametric test was applied. The Kruskal-Wallis test was used with a 0.05 significance level to verify differences between measurements. A significant difference with 95\% confidence interval was observed between the scores obtained in the initial and final evaluations that used the Tinetti [14] and EMTI [15] scales.

The study was approved by the Ethics Committee in Research including Human Beings from the School of Medicine of the Fluminense Federal University/FM/UFF/HUAP under number 531.807 in 2014. The ethical principles of voluntary and informed participation were respected.

\section{Results}

Table 1 shows results from the normality Shapiro-Wilkstest performed to verify the presence of normal distribution and determine the best statistical test to be used.

Because no variable other than the General Motor Ability (GMA) presented normal distribution, the nonparametric test signal was applied. All variables showed differences through the equality test with $95 \%$ confidence interval and 0.05 significance: that is, differences were observed between initial and final evaluation scores. These tests were not used for the MMSE evaluation because it was only conducted in the initial evaluation of cognitive impairment.

Table 2 shows the sociodemographic data and MMSE scores. The average age was 74.36 years, and the

Table 1. Normality and average similarity tests in the study variables. Rio de Janeiro, 2014.

\begin{tabular}{ccc}
\hline Variable & Normality test & Average similarity test \\
\hline MF1 (fine motricity) & 0.0001 & 0.0001 \\
MF2 (global motricity) & 0.0004 & 0.0106 \\
MF3 (balance) & 0.0015 & 0.0001 \\
MF4 (body scheme/agility) & 0.0001 & 0.0001 \\
MF5 (spatial organization) & 0.0001 & 0.0001 \\
MF6 (temporal organization) & 0.0001 & 0.0001 \\
GMF (general motor fitness) & 0.3042 & 0.0001 \\
Tinetti (balance and gait) & 0.0001 & 0.0001 \\
\hline
\end{tabular}

Source: Study datafrom 2014. MF = Motor Fitness.

Table 2. Distribution of elderlies according to gender, age, and MEEM scores. Rio de Janeiro, 2014.

\begin{tabular}{|c|c|c|c|c|c|}
\hline \multicolumn{4}{|c|}{ Variável } & $\mathbf{n}$ & $\%$ \\
\hline \multirow{2}{*}{ Gender } & \multicolumn{3}{|c|}{ Female } & 19 & 86.36 \\
\hline & \multicolumn{3}{|c|}{ Male } & 3 & 13.64 \\
\hline \multirow{3}{*}{ Age } & \multicolumn{3}{|c|}{$60-69$} & 5 & 22.73 \\
\hline & \multicolumn{3}{|c|}{$70-79$} & 12 & 54.54 \\
\hline & \multicolumn{3}{|c|}{$80-89$} & 5 & 22.73 \\
\hline \multirow{3}{*}{ MEEM } & \multicolumn{3}{|c|}{ Suggestive of cognitive deficit ( 23 points or less) } & 5 & 22.73 \\
\hline & \multicolumn{3}{|c|}{ Doubtful or alterations not suggestive of deficit (24 - 26 points) } & 8 & 36.36 \\
\hline & \multicolumn{3}{|c|}{ Preservedcognitivefunctions (27 - 30 points) } & 9 & 40.91 \\
\hline Variable & Average & Standard Deviation & Minimum & Maximum & Amplitude \\
\hline MEEM & 25.72 & 2.27 & 22 & 30 & 8 \\
\hline Age & 74.36 & 6.75 & 64 & 88 & 24 \\
\hline
\end{tabular}

Source: Study data from 2014. 
standard deviation was 6.75 . The ages ranged from 88 to 64 years old.

MMSE results averaged 25.72 points, ranging from 22 to 30 points. We observed $59.09 \%$ of participants with some cognitive deficit; $22.73 \%$ with a suggestive cognitive deficit, and $36.36 \%$ with alterations that were not suggestive of cognitive deficit. A total of $40.91 \%$ was observed with preserved cognitive functions. All selected participants remained in the study because they had been referred by geriatricians to the cognitive workshop due to memory shortage complaints.

Table 3 shows the results of the two evaluations using the Tinetti balance and gait scale.

According to Table 3, 31.82\% of the elderlies initially presented a high risk of falls, with $36.36 \%$ presenting a moderate risk of falls. After the intervention, only $9.09 \%$ presented a high risk of falls evaluation, with the majority, 59.09\%, presenting a moderate risk of falls. According to the Tinetti scale, $31.82 \%$ of the elderlies did not present a risk of falls in both evaluations.

Table 4 presents the results of the initial and final evaluations using the Motor Scale for the Elderly (EMTI). Fine Motricity (FM1) showed an average of 105 points in the initial evaluation, and 105.81 points in the final evaluation, both equivalent to the average normal classification.

Global Motricity (GM2) averaged 38.72 points in the initial evaluation, and 38.73 points in the final evaluation, corresponding to the extreme low classification.

Table 3. Distribution of elderlies according to scores obtained in the Tinetti Scale and results using the Kruskal-Wallis test. Rio de Janeiro, 2014.

\begin{tabular}{|c|c|c|c|c|c|c|c|c|}
\hline \multirow[b]{2}{*}{$\mathbf{n}$} & \multicolumn{4}{|c|}{ First evaluation } & \multicolumn{4}{|c|}{ Second evaluation } \\
\hline & $\%$ & Average & SD & Tinetti scale & $\mathbf{n}$ & $\%$ & Average & SD \\
\hline 7 & 31.82 & 17.42 & 1.14 & High risk of falls $(<19)$ & 2 & 9.09 & 17.5 & 0.70 \\
\hline 8 & 36.36 & 21.75 & 1.28 & Moderate risk (19 - 24) & 13 & 59.09 & 21.30 & 2.14 \\
\hline 7 & 31.82 & 26.86 & 1.46 & No risk of falls (>24) & 7 & 31.82 & 26.86 & 1.46 \\
\hline
\end{tabular}

Source: Study data from 2014.

Table 4. Distribution of variables according to motor areas and classification of scores in the EMTI scale, and results using the Kruskal-Wallis test. Rio de Janeiro, 2014.

\begin{tabular}{|c|c|c|c|c|c|c|c|c|}
\hline \multicolumn{5}{|c|}{ First evaluation } & \multicolumn{4}{|c|}{ Seconde valuation } \\
\hline Average & SD & \multicolumn{2}{|l|}{$P$ value } & EMTI scale & Average & SD & \multicolumn{2}{|c|}{ Pvalue } \\
\hline 105 & 13.57 & \multicolumn{2}{|l|}{0.001} & Fine motricity (MF1) & 105.81 & 12.40 & \multicolumn{2}{|c|}{0.002} \\
\hline 38.72 & 14.78 & \multicolumn{2}{|l|}{0.0483} & Global motricity (MF2) & 38.73 & 14.78 & \multicolumn{2}{|c|}{0.005} \\
\hline 89.18 & 26.72 & \multicolumn{2}{|l|}{0.0317} & Balance (MF3) & 92.73 & 22.66 & \multicolumn{2}{|c|}{0.009} \\
\hline 77.45 & 19.14 & \multicolumn{2}{|l|}{0.0001} & Body scheme/agility (MF4) & 82.91 & 16.93 & \multicolumn{2}{|c|}{0.0001} \\
\hline 109.63 & 19.95 & \multicolumn{2}{|l|}{0.0014} & Spatial organization (MF5) & 111 & 17.83 & \multicolumn{2}{|c|}{0.0006} \\
\hline 110.18 & 20.49 & \multicolumn{2}{|l|}{0.0003} & Temporal organization (MF6) & 110.72 & 19.91 & \multicolumn{2}{|c|}{0.0003} \\
\hline 88.36 & 10.26 & \multicolumn{2}{|l|}{0.0017} & General motorability (MFA) & 90.32 & 9.48 & \multicolumn{2}{|c|}{0.0007} \\
\hline \multicolumn{4}{|c|}{ First evaluation } & & \multicolumn{4}{|c|}{ Second evaluation } \\
\hline \multicolumn{2}{|c|}{ MF3 } & \multicolumn{2}{|c|}{ MFG } & EMTI scale & \multicolumn{2}{|c|}{ MF3 } & \multicolumn{2}{|c|}{ MFG } \\
\hline $\mathbf{n}$ & $\%$ & $\mathbf{n}$ & $\%$ & Classification & $\mathbf{n}$ & $\%$ & $\mathbf{n}$ & $\%$ \\
\hline 7 & 31.82 & 1 & 4.54 & Very low $(<69)$ & 1 & 4.54 & 0 & 0 \\
\hline 1 & 4.54 & 2 & 9.09 & Low (70 - 79) & 7 & 31.82 & 1 & 4.54 \\
\hline 4 & 18.18 & 12 & 54.54 & Normal low (80 - 89) & 4 & 18.18 & 9 & 40.90 \\
\hline 5 & 22.73 & 6 & 27.27 & Normal medium (90 - 109) & 5 & 22.73 & 11 & 50 \\
\hline 0 & 0 & 1 & 4.54 & Normal high (110 - 119) & 0 & 0 & 1 & 4.54 \\
\hline 2 & 9.09 & 0 & 0 & High (120 - 129) & 2 & 9.09 & 0 & 0 \\
\hline 3 & 13.63 & 0 & 0 & Very high (>130) & 3 & 13.63 & 0 & 0 \\
\hline
\end{tabular}

Source: Study data. SD—Standard deviation. 
An average of 89.18 points was observed in the initial evaluation of balance (MF3), equivalent to the normal low classification. An average of 92.73 points was observed in the final evaluation.

The Body Scheme/agility (MF4) averaged 77.45 points in the initial evaluation. The final evaluation averaged 82.91 points, corresponding to classifications of low and normal low, respectively.

The Spatial Organization averaged 109.63 points in the initial evaluation, equivalent to the normal average classification. The final evaluation averaged 111 points corresponding to the normal high classification.

The Temporal Organization averaged 110.18 points, equivalent to the normal high evaluation. The final evaluation averaged 110.72 points, corresponding to the normal high classification.

Table 4 shows General Motor Fitness (GMF) results, an average obtained from the sum of all motor areas in the EMTI scale. Most elderlies (54.55\%) obtained scores below 89 and were initially classified as normal low. Six elderlies (27.27\%) were classified in the normal average classification with scores between 90 and 109; two elderlies (9.09\%) with scores lower than 79 were classified as low. One elderly with scores lower than 69 was classified as very low. One elderly scored between 110 and 119 and was classified as normal high. The average GMF in the first evaluation was 88.36 points.

The analysis using the Kruskal-Wallis test with 95\% confidence interval and 0.05 level of significance showed that all motor fitness (MF), except Global Kinetics and Motricity (MF2), which presented 95.45\% of elderlies in the classification of very low and $4.55 \%$ as low, were significantly different between the initial and final evaluations.

\section{Discussion}

The predominance of women in the study (86.36\%) is similar to that reported in other studies and may be related to male higher mortality rates. [16] The increased demand for health services by women mainly due to gender factors and related to culture [17] may also be a contributing factor. A total of $54.54 \%$ of participants were aged between 70 and 79 years old.

The Mini-Mental State Examination results (Table 1) show that the vast majority (59.09\%) of elderlies presented some type of cognitive impairment, $22.73 \%$ demonstrated signs suggestive of cognitive impairment with scores ranging from 22 to 23 points, and $36.36 \%$ exhibited either questionable changes or no suggestive cognitive deficit. This result is supported by another study in the elderly population where $34.1 \%$ shows cognitive impairment [18].

The MMSE evaluation shows the diagnosis of impaired memory, which is understood as the inability to remember or retrieve information or behavioral skills [10]. Participants were advised about the benefits of an early diagnosis and the importance of participating in psychomotor activities.

Psychomotor activities were organized as either individual or joint participation. A willingness to increased communication [10] was observed as the elderlies interacted well not only with the professional guiding the activities but also with each other. Participants gave and received suggestions, and exchanged information in order to reach goals, demonstrating a willingness to increased decision-making [10]. It is important to promote the elderlies' input to demonstrate that their needs are valued and to prevent evasion.

The risk of falls [10] was observed in the diagnosis of Perception/Control of health in the initial evaluation assessing balance and gait using the Tinetti scale and the balance parameter (MF3) using the EMTI scale. In the results from the Tinetti scale, $68.18 \%$ of the elderlies showed some changes in balance and aspects related to difficulty in gait, such as the length and height of steps, trunk position, and distance between ankles. Some of the elderlies reported tripping over their feet, which indicated the need for interventions; 31.82\% showed a 17.42 point average, which corresponds to a high risk of falls, and $36.36 \%$ showed the moderate risk of falls with an average of 21.75 points.

The comparison of balance and gait using the Tinetti scale with the motor balance parameter (MF3) using the EMTI scale showed that $31.82 \%$ of the elderlies presented scores lower than 69 points in the EMTI scale, corresponding to the classification of very low in the first evaluation. The average obtained in this parameter by the studied participants was 89.18 points and were classified as normal low in the first evaluation.

The results using the Tinetti scale in the final evaluation showed that $22.73 \%$ of the elderlies were able to improve balance and gait, and 59.09\% moved from high to moderate risk of falls. Despite continuing at moderate risk of falls, 36.36\% of participants showed an increase in their scores. However, 31.82\% of the elderlies maintained an average of 26.86 points, suggesting no risk of falls in either evaluation. A total of $27.27 \%$ of the elder- 
lies obtained improved scores in the balance parameter (MF3) in the final evaluation, improving their classification from very low to low. The average of participants was 92.73 points, and their classification changed to normal average in the EMTI scale.

The score increase in balance and gait in the Tinetti scale supports this result because the results from the two scales used in the second evaluation showed satisfactory improvement in balance. Balance is formed by a set of static and dynamic skills including postural control and development of motor fitness; it is considered as the primary basis for all differentiated action of the body and is central to coordination [15].

The diagnostic evaluation using the Motor Scale for the Elderly-EMTI, which features six motor parameters (Table 4), shows Fine Motricity (MF1) scores between 90 and 109 with an average of 105 points, classified as normal average in the first evaluation; the average for the second evaluation was 105.81 points. Despite the unchanged fine motricity, the average increased. The precentral cortex corresponding to the fine motricity skills plays a key role in the control of isolated movements of hands and fingers. The cortico-sensorial motor areas of hands and fingers are relevant because they emphasize the finesse of tactile and motor controls [16].

Although numerical scores modestly increased in the second evaluation, twenty-one elderlies (95.45\%) presented final classification as very low in the EMTI scale for global motricity (MF2). Most of the motor parameter test proves difficult for those who do not practice physical activities because they include climbing on a bench, jumping over a rope, jumping in place, jumping at $20 \mathrm{~cm}$ height, walking on a straight line, doing the "soft foot" jump at $40 \mathrm{~cm}$ height, jumping in the air, doing the "soft foot" with a box of matches, and jumping over a chair [15].

Therefore, the diagnoses according to NANDA [11] show sedentary lifestyle and deficient recreational activities. Elderlies who do not attend a gym or establishments that provide physical fitness do not feel safe to carry out such tests; it is understood that with the aging process and progressive decrease in muscle strength, muscle tone also decreases [4].

The comparison of results from fine motricity, global motricity, and balance showed that fine motricity presented the most satisfactory results because the obtained scores classified the elderlies as normal average. The global motricity parameter (MF2) showed the highest degree of difficulty out of all evaluated motor areas and was classified as very low in both evaluations. This is because this area is responsible for regulating balance and the individual's attitude, playing an important role in the improvement of nerve commands and refining of perceptions and feelings [15].

The comparison between the motor parameters of body scheme (MF4), spatial organization (MF5), and temporal organization (MF6) showed that spatial organization (MF5) was the criterion that received the highest scores in the final evaluation; it was classified as normal high as opposed to normal average in the initial evaluation. The elderlies were classified as normal high in both evaluations of temporal organization (MF6). They showed the lowest scores in body scheme (MF4); however in the second evaluation, they showed improved scores and moved from the classification of low to normal low. These increased scores indicated an improvement in these parameters.

The movements considered most difficult for the elderly are those of body dissociation, lateral domain, coordination of upper and lower limbs, speed, agility, breath, proprioception, rhythm, and body memory [16]. Such movements are primarily associated with the natural aging process as well as physical exercise, motivation, and quality of life [4].

The Motor Scale for the Elderly (EMTI) presented the General Motor Fitness (GMF) measurements according to the scores obtained in the initial and final evaluation and showed the effectiveness of activities and satisfactory improvement in the evaluated motor parameters.

It is important to diagnose perceived needs early and, from there, guide the elderly in a continuous and effective way, including benefits provided by regular physical exercises, to prevent and treat functional declines associated with the aging process.

\section{Conclusions}

The use of the MMSE, Tinetti, and EMTI Scales allowed the diagnoses of psychomotor deficits, which were observed among perceived needs. The results from the use of the Tinetti scale showed that $68.18 \%$ of the elderlies were associated with risk of falls, $31.82 \%$ were at high risk of falls, and $36.36 \%$ at moderate risk of falls. The results from the use of the EMTI scale showed low scores in the parameters of global motricity, balance, 
and body scheme/speed in the initial evaluation, and increased scores in the final evaluation. The identified diagnoses were a risk of falls, sedentary lifestyle, and deficient recreational activities.

The elderlies showed improved performance in the temporal organization, spatial organization, and fine motricity areas. The EMTI scores were 83.11 points (normal low classification), and 90.72 points (normal average classification) in the first and second evaluations, respectively. This result demonstrates the satisfactory effect of interventions.

The results from the use of MMSE showed that 59.09\% of the elderlies had some cognitive impairment, and 22.73\% had signs suggestive of cognitive impairment and diagnosis of impaired memory. Elderlies who participated in the activities demonstrated increased communication, enhanced decision making, and improved relations.

This study contributes significantly to the development of strategies to care for the elderly with mild cognitive impairment by improving functional capacity.

\section{References}

[1] Instituto Brasileiro de Geografia e Estatística (2010) Censo 2010: População do Brasil é de 190.732.694 Pessoas. http://censo2010.ibge.gov.br/pt/noticias-censo?view=noticias\&id=3\&pagina=3\&paginar=1\&quantidade=10\&busca=1

[2] Antonini, F.M, et al. (2008) Physical Performance and Creative Activities of Centenarians. Archives of Gerontology and Geriatrics, 46, 253-261. http://dx.doi.org/10.1016/j.archger.2007.04.005

[3] Rabelo, D.F. (2009) Comprometimento cognitivoleve em idosos: Avaliação, fatoresassociados e possibilidades de intervenção. Revista Kairós Gerontologia, São Paulo (SP), 12, 65-79.

[4] Costa, M., Rocha, L. and Oliveira, S. (2012) Educação em saúde: Estratégia de promoção da qualidade de vida naterceira idade. Revista Lusófona de Educação, Lisboa, 22, 123-140. http://revistas.ulusofona.pt/index.php/rleducacao/article/viewFile/3285/2434

[5] Petersen, R.C., Caracciolo, B., Bryne, C., Gauthier, S., Jelic, V. and Fratiglioni, L. (2014) Mild Cognitive Impairment: A Concept in Evolution. Journal of Internal Medicine, 275, 214-228.

[6] Canineu, P.R., Samara, A.B. and Stella, F. (2011) Transtorno Cognitivoleve. In: Freitas, E.V., et al., Eds., Tratado de geriatria e gerontologia, 3rd Edition, Guanabara Koogan, Rio de Janeiro, Vol. 14, 170-177.

[7] Marinho, L.M., Vieira, M.A., Costa, S.M. and Andrade, J.M.O. (2013) Grau de dependência de idosos residentes em instituições de longa permanência. Revista Gaúcha de Enfermagem, 34, 104-110. http://dx.doi.org/10.1590/S1983-14472013000100013

[8] Reis, L.A., Reis, L.A. and Torres, G.V. (2015) Impacto das variáveis sociodemográficas e de saúde na Capacidade funcional de idosos de baixa renda. Ciência, Cuidado e Saúde, 14, 847-854.

[9] Fuhrmann, A.C., Bierhals, C.C.B.K., Santos, N.O. and Paskulin, L.M.G. (2015) Associação entre a capacidade funcional de idosos dependentes e a sobrecarga do cuidador familiar. Revista Gaúcha de Enfermagem, 36, 14-20. http://dx.doi.org/10.1590/1983-1447.2015.01.49163

[10] Chaves, L.D. and Solai, C.A. (2013) Sistematização da Assistência de enfermagem Considerações Teóricas e aplicabilidade. Ed. 2. Martinari, São Paulo.

[11] Doenges, M.E., Moorhouse, M.F. and Murr, A.C. (2013) [Tradução Cosendey CH] Diagnóstico de Enfermagem: Intervenções, prioridade, fundamentos. Guanabara Koogan, Rio de Janeiro.

[12] Folstein, M.F., Folstein, S.E. and Mchugh, P.R. (1975) Mini-Mental State: A Practical Method for Grading the Cognitive State of Patients for the Clinician. Journal of Psychiatric Research, 12, 189-198.

www.ncbi.nlm.nih.gov/pubmed/1202204 http://dx.doi.org/10.1016/0022-3956(75)90026-6

[13] Bertolucci, P.H., Bruck, S.M., Campacci, S. and Juliano, Y. (1994) The Mini-Mental State Examination in a General Population: Impact of Educational Status. Arquivos de Neuro-Psiquiatria, 52, 1-7. http://www.ncbi.nlm.nih.gov/pubmed/8002795 http://dx.doi.org/10.1590/S0004-282X1994000100001

[14] Tinetti, M.E. (1986) Performance-Oriented Assessment of Mobility Problems in Elderly Patients. Journal of the American Geriatrics Society, 34, 119-126. http://dx.doi.org/10.1590/S0004-282X1994000100001

[15] Neto, F.R., et al. (2009) Manual de Avaliação Motora para Terceira Idade. Artmed, Porto Alegre.

[16] Neto, F.R., Sakae, T.M. and Poeta, L.S. (2011) Validação dos parâmetros motores na terceira idade. Revista Brasileira de Ciência e Movimento, 19, 20-25.

[17] de Lima, E.H. ( 2012) Gênero, masculinidades, juventudes e uso de drogas: Contribuições teóricas para a elaboração de 
estratégias em educação em saúde. Pesquisas e Práticas Psicossociais, 7, 279-289.

[18] Holz, A.W., Nunes, B.P., Thumé, E., Lange, C. and Facchini, L.A. (2016) Prevalence of Cognitive Impairment and Associated Factors among the Elderly in Bagé, Rio Grande do Sul, Brazil. Revista Brasileira de Epidemiologia, 16, 880888. http://www.scielo.br/scielo.php?script=sci_arttext\&pid=S1415-790X2013000400880\&lng=en

http://dx.doi.org/10.1590/S1415-790X2013000400008

\section{Submit or recommend next manuscript to SCIRP and we will provide best service for you:}

Accepting pre-submission inquiries through Email, Facebook, LinkedIn, Twitter, etc.

A wide selection of journals (inclusive of 9 subjects, more than 200 journals)

Providing 24-hour high-quality service

User-friendly online submission system

Fair and swift peer-review system

Efficient typesetting and proofreading procedure

Display of the result of downloads and visits, as well as the number of cited articles

Maximum dissemination of your research work

Submit your manuscript at: http://papersubmission.scirp.org/ 